\title{
Shell Part of Nucleus Accumbens and Its Laterality Has Important Role in Response to Chronic Stress in Female Rats
}

Tahereh-Sadat Javadifar ${ }^{1}$

https://orcid.org/0000-0002-5203-2624

\section{Mehdi Amirikia ${ }^{3}$}

https://orcid.org/0000-0002-3180-8809

Nahid Sarahian ${ }^{1}$

https://orcid.org/0000-0002-7915-7355

\author{
Hedayat Sahraei ${ }^{1^{*}}$ \\ https://orcid.org/0000-0001-9235-0013
}

\author{
Mohammad Nasehi ${ }^{5}$ \\ https://orcid.org/0000-0002-1981-3592 \\ Mohammad-Reza Zarrindast ${ }^{4}$ \\ https://orcid.org/0000-0002-0688-5378
}

\section{Mohammad-Ali Ketabi ${ }^{2}$ \\ https://orcid.org/0000-0002-9800-0038}

${ }^{1}$ Baqiyatallah University of Medical Sciences, Neuroscience Research Center, Tehran, Iran; ${ }^{2}$ Aja University of Medical Sciences, School of dentistry, Department of Endodontics, Tehran, Iran; ${ }^{3}$ Babol University of Medical Sciences, Health Research Institute, Fatemeh Zahra Infertility and Reproductive Health Research Centre, Babol, Mazandaran, Iran; ${ }^{4}$ Tehran University of Medical Sciences, School of Medicine, Department of Pharmacology, Tehran, Iran; Islamic Azad University, CNRC,Tehran Medical Science Branch, Cognitive and Neuroscience Research Center, Tehran, Iran.

Received: 2019.05.23; Accepted: 2020.02.12.

${ }^{*}$ Correspondence: h.sahraei@bmsu.ac.ir; Tel.: (9821) 26127286, Baqiyatallah University of Medical Sciences, Neuroscience Research Center, Tehran, Iran.

\section{HIGHLIGHTS}

- Chronic stress increase food intake and blood glucose significantly.

- Chronic stress has no effect on delay to start eating and amount of water intake.

- $\quad$ Right side and left side of NAcSh are dominant in delay and amount of food intake.

- Stress increase estradiol level but has no effect on corticosterone and progesterone.

Abstract: The nucleus accumbens shell (NAcSh) plays a role in appetitive and negative motivation with sex differences in responses. NAcSh and its laterality in metabolic and hormonal responses to chronic stress in female rats is evaluated via transient inactivation of this nucleus during stress induction. Animals in the stress groups received consecutive stress for four days and transient inactivation of NAcSh was performed by administrating lidocaine $(0.2 \%)$ unilaterally or bilaterally in the nucleus for five minutes before electric foot shock induction. After stress termination, food and water intake, latency to eat, plasma glucose, corticosterone, estradiol and progesterone were measured in all groups. Results showed that stress increased food intake and blood glucose level, but there were no change in the latency to eat and the amount of water intake. The right side, the left side, and both sides of NAcSh may be dominant in latency to eat, food intake, and both water intake and plasma glucose level, respectively. Although chronic stress included no changes for corticosterone and progesterone, it increased estradiol level in plasma. Also, bilateral and right sides of NAcSh may have modulatory effects on stress in corticosterone and progesterone, respectively, 
without affecting estradiol. It can be concluded that the NAc shell plays a pivotal role in metabolic and hormonal responses to chronic stress in a laterality manner in female rats.

Keywords: chronic stress; female rats; Lidocaine; Nucleus Accumbens Shell; laterality.

\section{INTRODUCTION}

Nowadays, many human beings are exposed to psychological and physical stress that subsequently results in various diseases such as gastric ulcer, hypertension, hyperthermia and affective disorders. Hence, there is significant increase in psychological and physical stress. Stress can be divided in chronic and acute stages, which can lead to behavioral and physiological changes in animals [1-9]. Chronic stress is considered the cause of maladaptive responses that harm neuroplasticity and finally result in cognitive and behavioral deficiency [10-11]. Therefore, chronic stress as a post-traumatic stress disorder (PTSD), major depressive disorder (MDD) and anxiety is involved in the pathophysiology of neuropsychiatric disorders [12]. It is shown that females are more vulnerable to stress-induced anxiety and depression [13]. Various studies also demonstrated that there are many gender based differences in molecular and cellular substrates of stress [14]. However, a few studies consider the role of the shell part of the nucleus accumbens (NAcsh) in the metabolic and hormonal responses to chronic stress.

The hypothalamic-pituitary-adrenal (HPA) axis is an important factor that is responsible for the adaptive response in the face of physical or psychological stress. After exposure to stressful stimuli, a series of hormones including corticotrophin-releasing factor (CRF), adrenocorticotropic hormone, and glucocorticoids (GCs) are released from the paraventricular nucleus of the hypothalamus, anterior pituitary, and adrenal cortex, respectively [15-16]. These affect the physiology throughout the organism, including the CNS [17]. Multiple compartments of brain including ventral tegmental area, prefrontal cortex, amygdala, and hippocampus may be affected by stress hormones released in the central nervous system following the exposure to unavoidable, aversive stimuli and the changes in related neuron physiology. It is possible that the nucleus accumbens (NAc) function, a motor-limbic interface related to reward and motivation, might also be altered in response to stress, as it receives inputs from each of those stress-affected brain areas. For example, in the study of Gill and coauthors in 2013, the impact of acute and repeated stress on the integration of context and emotion inputs in the nucleus accumbens was demonstrated [18].Previous research revealed that stress inducing leads to elevated dopamine utilization in shell part of NAc [19] which shows changes in NAcsh after the induction. Moreover, the right and left sides of NAc differently connect to other regions of the CNS [20-21]. Functional and anatomical asymmetry have also been observed between the right and left sides of the NAc [22-23]. In our previous study, it was demonstrated that the NAc shells asymmetrically plays an important role in mediating hormonal and metabolic responses in acute foot-shock stress in female rats [24]. However, there is insufficient data to identify the modulatory role of NAc shell in metabolic and hormonal factors in chronic stress. In this study, we defined the significant role of left, right or both sides of NAc shell is discussed in hormonal responses (changes in corticosterone, progesterone and estradiol concentrations), blood glucose and metabolic responses (food and water intake and latency to eat) in female rats exposed to chronic stress. Therefore, intra accumbal Lidocaine was administrated to inactivate the NAc shell sides during stress induction.

\section{MATERIAL AND METHODS}

\section{Animals}

Forty-five female animals (Wistar rats, W: $200 \pm 20 \mathrm{~g}$, Pasture Institute, Tehran, Iran) were kept in cages (4/cage) at a temperature of $(22 \pm 2)^{\circ} \mathrm{C}$, and a 12 hours light, 12 hours dark cycle. The rats had free access to tap water and standard feed (standard pellets, Pars Production and Distribution of Animal Feed Company, Iran).Animals received intra-NAc injections of $2 \%$ Lidocaine solution either bi- or unilaterally before stress ( $n=7$ for right side group, $n=7$ for left side group and $n=8$ for bilateral groups, that all of them received lidocaine). The sham group $(n=7)$ received normal saline and control group $(n=8)$ did not receive any treatment. The camera system was applied to record animal's food and water intake during days and nights. All procedures were approved by the Animal Care and Use Committee of the Neuroscience Research Center, Baqiyatallah (a.s) University of Medical Sciences, Iran. 


\section{Drugs}

To inactivate the sell parts of NAc, Lidocaine hydrochloride (Sigma, St. Louis, MO, USA) with a dose of $2 \%$ prepared by sterile saline [25] and at a volume of $0.25 \mu \mathrm{L} /$ side was administered in intra-NAc 5 minutes before inducing stress. To anesthetize ketamine hydrochloride $(50 \mathrm{mg} / \mathrm{kg}$,ip) and diazepam ( $5 \mathrm{mg} / \mathrm{kg}$,ip) (both from Sigma, St. Louis, MO, USA) were prepared at a concentration of $50 \mathrm{mg} / \mathrm{mL}$ immediately before each surgery and then were injected intraperitoneally and at a volume of $1 \mathrm{~mL} / \mathrm{kg}$ to the animals.

\section{Stress induction}

An instrument (Borje Sanat Azma Corporation, Tehran, Iran) was applied to induce shock in animals in a standard condition [26]. Briefly, the instrument had nine distinct Plexiglas chambers $(16 \times 16 \times 50 \mathrm{~cm}$, length $\times$ width $\times$ height). There were parallel rods $(4 \mathrm{~mm}$ in diameter) with a distance of $1.3 \mathrm{~cm}$ from each other on the floor of each chamber (Figure 3). The rods were connected to the generators controlled by software to produce an electrical current of $1 \mathrm{~mA}$ with $10 \mathrm{~Hz}$ frequency for $60 \mathrm{~s}$ randomly. Therefore, each animal received 600 electrical stimuli under randomized generator controlling during 100s that generator was activated. The test was performed every day for four days. In this study, non-continuous stress was applied to prevent adaptation of rats to uninterrupted electric foot shock and therefore the stress was new to animals every time they received it. In this study, nine-chambered instrument gave us the chance to test all the rats belonging to one group ( $n=7-8)$ simultaneously. Clear Plexiglas of chambers prepared conditions that allowed each rat to see and hear other rats getting shocked. All of the aforementioned conditions were the same among the sham and experimental groups.

\section{Identification of Estrous cycle}

Animal's estrous phase was examined for four days before the stress day. Briefly, vaginal smear test was applied each morning between 8:00 and 9:00 a.m. Then smears were observed by light microscopic to determine the estrous phase. Although animals were in different phases of estrous, those in proestrous cycle were chosen for the experiments, as shown in Figure1. This was due the fact that, plasma estradiol level reaches its highest level in this phase [27]. In addition to estradiol, plasma corticosterone also is in its high level, since the HPA axis becomes more sensitive in proestrous cycle [28].

\section{Experimental design}

To inactivate NAc either bi-or unilaterally, $2 \%$ Lidocaine solution was injected into intra-NAc before stress ( $n=8$ for each side group and $n=8$ for bilateral groups). Normal saline was injected to the sham $(n=8)$ and control groups $(n=8)$ that did not receive any treatment. Electro foot shock was applied to each group once (experiments performed between 9:00 and 16:00). To adapt animals to the experimental environment, they were kept in an experimental room for an hour and then in the compartments (one per compartment) for 30 minutes before the experiment onset. All groups, except control groups, were induced by electro foot shock during 100 seconds. Next, the animals were left in the compartment for an additional 30 minutes, and then were returned to their home cages. The induction of stress was repeated every day for four days.

\section{Metabolic measurement}

During the first 24 hours after the last stress sessions on the fourth day, food and water intake by each rat were controlled and measured in terms of the percentage of food and water consumption. The latency to eat (the time elapsed before the animals started to eat chow after being returned to their cages) was also recorded as an index of stress.

\section{Hormonal measurement}

Immediately 30 minutes after the last experiment on the fourth day, blood samples were taken from the animals' retro-orbital sinus to measure hormones changes. $1 \mathrm{~mL}$ of blood was mixed with $0.1 \mathrm{~mL}$ of $3 \%$ EDTA solution and was centrifuged at $3000 \mathrm{~g}$ for 7 minutes at $4{ }^{\circ} \mathrm{C}$ to isolate plasma, and then was kept at $-20^{\circ} \mathrm{C}$ up until it was used for corticosterone, estradiol and progesterone measurement (Toleikis and Godin, 1995). ELISA kits including Rat Corticosterone ELISA kit; EIA-4164; DRG Instruments GmbH, Germany, and Diagnostics Biochem Canada Inc were used to determine corticosterone, estradiol, and progesterone concentrations at $450 \mathrm{~nm}$, respectively. Coefficients of variation percent (CV\%) for control samples supplied by the manufacturers, were as follows: the intra-assay CV for three samples $(n=12)$ of corticosterone 
hormone included $3.09 \%$ and the inter-assay CV for two samples of progesterone hormone which were assayed 10 times each on the same calibrator curve included $10.4 \%$, and also the inter-assay CV for three samples of estradiol hormone which were assayed 10 times each on the same calibrator curve included $7.7 \%$.

\section{Surgical procedure}

After anesthetizing Animals by a solution containing ketamine hydrochloride $(50 \mathrm{mg} / \mathrm{kg}, \mathrm{i} . \mathrm{p})$ and Diazepam (5mg/kg,i.p), one or two stainless steel cannula (23gauges) were placed stereo- toxically (Stoelting Instruments, USA) in to the shell part of NAcuni or bilaterally and $0.5 \mathrm{~mm}$ above the intended site of injection, according to Paxinos and Watson (2007). The coordinates for the shell part of NAc were as follows: incisor bar $(-3.3 \mathrm{~mm}), 1.68 \mathrm{~mm}$ anterior to the bregma, $\pm 0.6 \mathrm{~mm}$ lateral to the sagittal suture, and $6.8 \mathrm{~mm}$ below the top of the skull Jeweler's screws with dental acrylic cement were used to secure cannula. After 5-7 days of recovery from surgery and anesthesia, the animals were used in the experiments. To inject NAc shells, animals were gently restrained by hands. Then a 29-gauge injection needle was placed in the guide cannula exactly $0.5 \mathrm{~mm}$ below the tip and then a total volume of $0.25 \mu \mathrm{L} /$ side Lidocaine of $2 \%$ solution was manually injected over a period of 60 seconds. Injection needle was not removed from its place for an additional 60 seconds up to the complete diffusion of the drug. Analgesic or antibiotic were not applied in this experience in order to prevent possible interferences of the drugs or their metabolites with the experimental procedure.

\section{Histology}

Histological test was used to screen animals for correct cannula placements. After animals' anesthesia by diethyl ether, $0.1 \mu \mathrm{L}$ of $1 \%$ aquatic methylene blue, as an ink, was infused into the injection site to facilitate histological identification. Trans cardiac perfusion of $0.9 \%$ normal saline followed by $4 \%$ buffered formalin was applied to block brains. Then, the brains were removed and cut coronally in $1 \mathrm{~mm}$ sections through the cannula placements (between 0.6 and $3.6 \mathrm{~mm}$ AP). The sections were observed using light dissection microscopy to confirm the correctness of cannula placements. Only the animals with correct cannula placements were included in the data analysis (Figure 2).

\section{Data analysis}

All the data are presented using mean \pm SEM. Statistical differences $(P<0.05)$ between groups were analyzed using one-way analysis of variance (ANOVA) once to evaluate the effect of stress as an independent variable and again to evaluate drug effect as another factor, followed by the Tukey post hoc test.

\section{RESULTS}

\section{Latency to eat}

The time it takes for the animals to begin eating after returning to their home cage is considered as the latency to eat and also as an index to evaluate metabolic response to the chronic stress in blocked NAc shell rats. The results showed that although chronic stress resulted in a slight decrease in the latency to eat, transient inactivation of the right and bilateral sides of shell part of NAcusing $2 \%$ Lidocaine increased the latency to eat significantly $[\mathrm{F}(4,30)=77.66, \mathrm{P}<0.001]$. Lidocaine administration in the left side of NAc shell did not show any effect on the latency to eat. Interestingly, the absence of the right side of the nucleus confirmed its dominant effect in bilateral interaction between the two sides of this nucleus in the stress condition of latency to eat (Figure $3(A)$ ).

\section{Food and water intake}

Studying the changes in food intake in the absence of NAc shell after chronic stress induction showed that food intake was significantly increased through chronic stress in animals $[F(4,30)=49.87, P<0.001]$. Lidocaine injection into the left side of the NAc shell enhanced the effect of stress on food intake in the experimental group in comparison with the saline-stressed group $[P<0.001]$ (Figure 3(B)).Based on the results, evaluating the effect of the NAc shell inactivation on water intake following chronic stress showed that the four-day stress decreased water intake, though this reduction was not statistically significant. In the experimental group, Lidocaine significantly augmented the stress effect on water intake when injected into 
the right, left, and/or bilateral sides of the NAc shell in comparison with the control and also saline-stressed groups $[\mathrm{F}(4,30)=70.68, \mathrm{P}<0.001]$ (Figure $3(\mathrm{c}))$.

\section{Blood glucose concentration}

The results of this experiment revealed that chronic stress could significantly elevate plasma glucose concentration in all the rats in saline group and also transient inactivated right, left, and/or bilateral sides of the NAc shell groups in comparison with non-stressed rats $[F(4,23)=37.89, P<0.001]$. According to Figure 4, uni-or bilateral of Lidocaine injection in to the NAc shells led to a significant increase in blood glucose concentration of the experimental groups in comparison with saline group $(P<0.001)$ (Figure 4$)$.

\section{Hormones concentration}

After four days of stress, the blood samples were collected to evaluate the effects of chronic stress on the plasma levels of corticosterone, progesterone, and estradiol hormones in the rats that received transient inactivation of NAc shell, animal. The results showed that chronic stress led to non-significant changes in corticosterone level in female rats. However, transient inactivation of NAc shell in the right side resulted in significant increase in plasma corticosterone concentration in comparisons with non-stress $(P<0.05)$ and saline stressed groups [F $(4,26)=5.42, \mathrm{P}<0.01]$. In addition, left-side inactivated group demonstrated enhanced corticosterone hormone compared to the saline- stressed group $[P<0.05]$, while there was no significant change in corticosterone level in bilateral NAc shell inactivated group (Figure 5(A)). Evaluations of plasma level of progesterone revealed that chronic stress could not change this hormone significantly. In contrast, transient inactivation of the NAc shell in the right-side resulted in significant increase in progesterone concentration versus non-stress and saline-stress groups $[F(4,25)=7.84, P<0.001][P<0.01]$. However, transient inactivation of NAc shell in the left and bilaterally sides displayed no significant change compared to the control groups (Figure 5(B)). Although chronic stress elevated estradiol concentration in plasma [F (4, 23) $=3.05, P<0.001]$, transient inactivation in the right, left and bilateral sides of NAc indicated no significant change compared to the control groups (Figure $5(\mathrm{C})$ ).

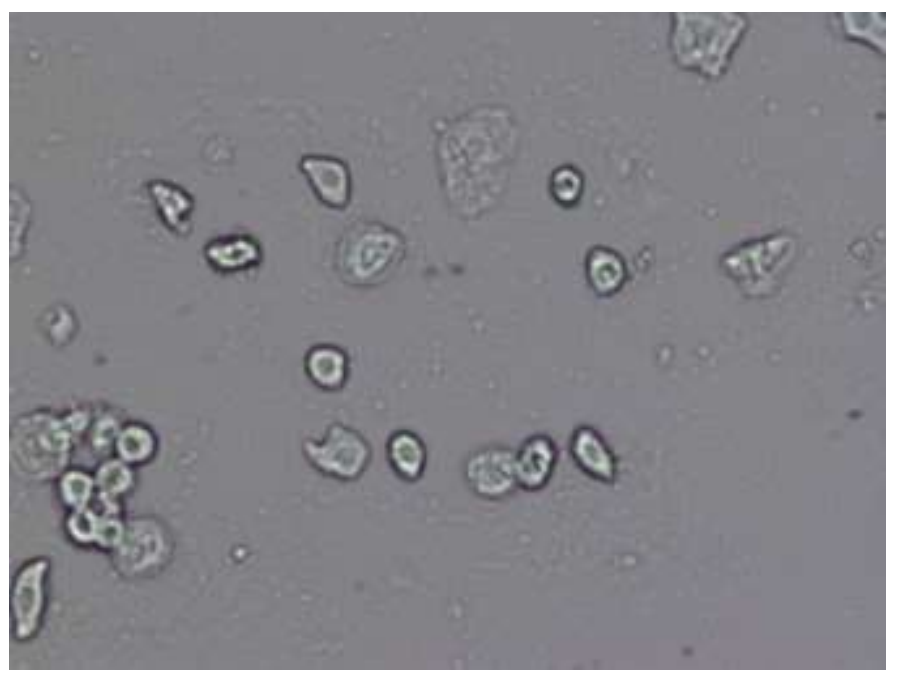

Figure 1. Determination of the Proestrus phase in estrous cycle in female rats. This phase consists of a predominance of nucleated epithelial cells. 


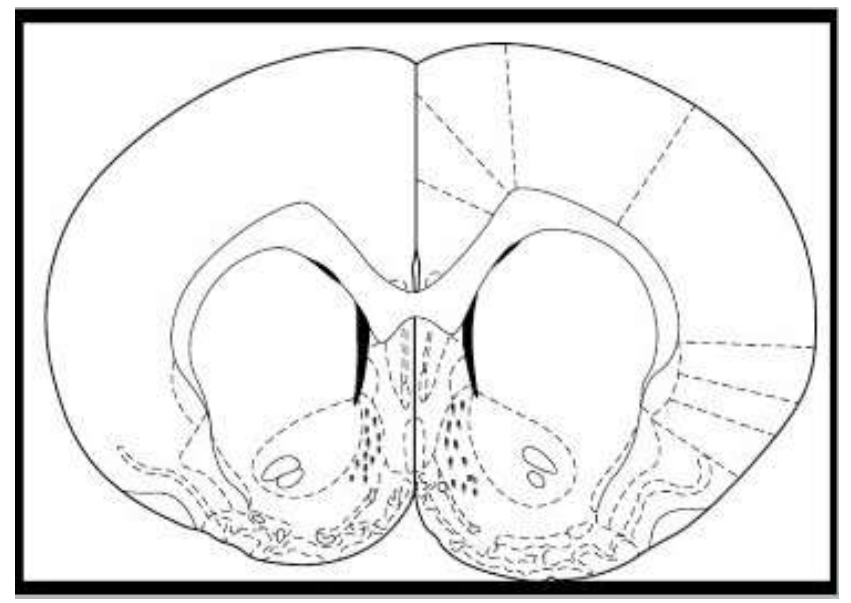

Figure 2. The signed areas with ticker lines in the shape are the illustration of cannula tip position in the NAc shell of female rats that used in our experiments. With (I) this sign.
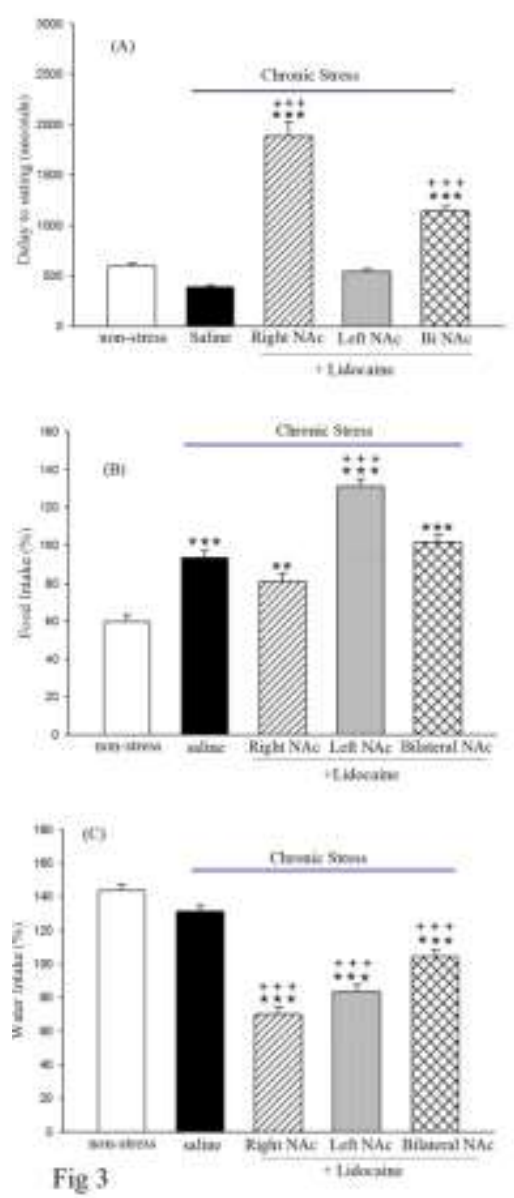

Figure 3. Effects of left, right, and/or bilateral transient inhibition of the NAc shell on the latency to eat (A), food intake $(B)$, and water intake $(C)$ after stress induction. Each column shows the mean $\pm S E M$ of time elapsed until animals began to eat after returning to their home cage $(A)$ and percentage score of food or water intake $24 \mathrm{~h}$ after four days of stress induction $(B, C)$ for $7-8$ rats. ${ }^{* *} P<0.001$, , ${ }^{* *} P<0.01$, different from the non-stress group; $+++P<0.001$, different from the saline-stress group. 


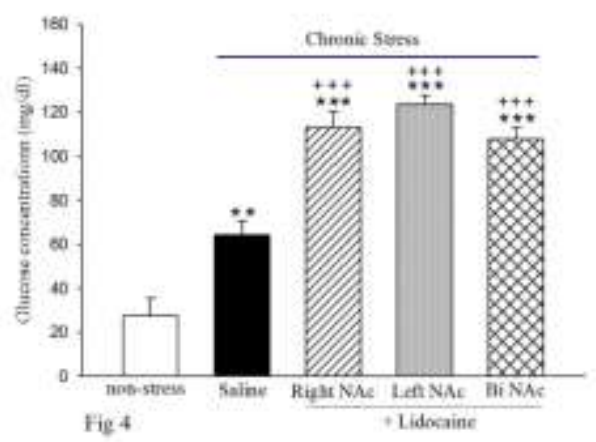

Figure 4. Effect of chronic stress on blood glucose levels in different groups. Blood glucose level was enhanced significantly by chronic stress. As figure shows, transient uni- or bilateral blocking of NAc shells by Lidocaine showed a significant increase $(P<0.001)$ of blood glucose levels in compression with the saline group. Each column shows the mean \pm SEM score of glucose concentration for $7-8$ rats. ${ }^{* * *} \mathrm{P}<0.001,{ }^{* *} \mathrm{P}<0.01$ different from non-stress group; +++ $\mathrm{P}<0.001$, different from the saline-stress group.
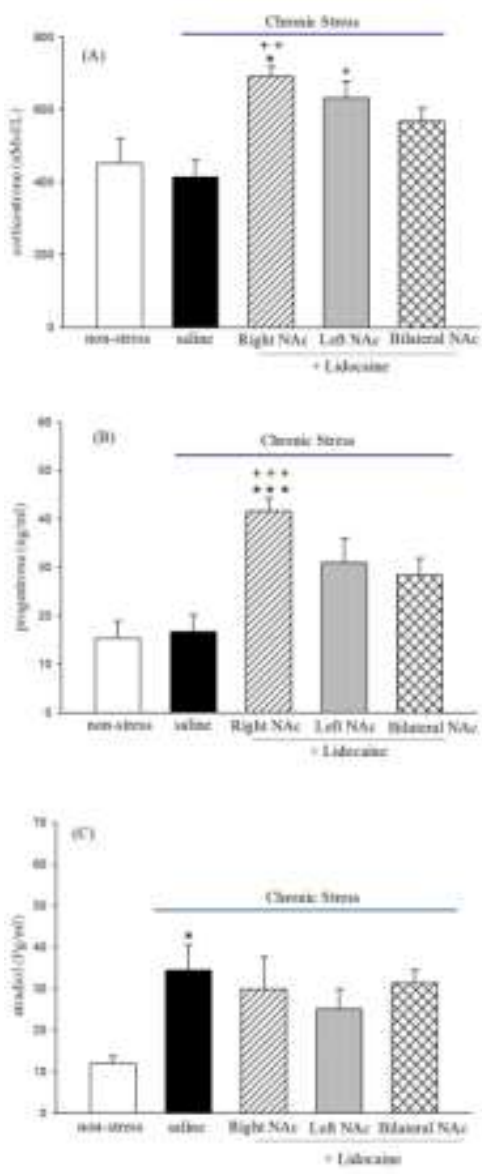

Fig 5

Figure 5. Effects of transient inactivation of left, right, and/or bilateral of the NAc on corticosterone (A), progesterone $(B)$, and estradiol $(C)$ immediately after the induction of stress. Each column shows the mean $\pm S E M$ score of hormone concentration for $7-8$ rats. ${ }^{* * *} \mathrm{P}<0.001,{ }^{*} \mathrm{P}<0.05$ different from the non-stress group; $+++\mathrm{P}<0.001,++\mathrm{P}<0.01,+\mathrm{P}<0.05$ different from the saline-stress group.

\section{DISCUSSION}

The main goal of this study was to evaluate the role of shell part of the nucleus accumbens and different tasks of both sides of this part of the brain structures in some metabolic and hormonal responses to chronic stress in female rats. Four parameters were tested including the food and water consumption, the concentration of blood glucose, and latency to eat after stress termination for evaluating the role of NAc shell 
in metabolic responses to chronic stress. Our finding demonstrated that chronic stress did not have any effect on the latency to eat and the amount of water intake, while it caused a remarkable increase in food intake and glucose concentration. In stress condition laterally inactivation of NAc shell(s) showed that the dominant side in the latency to eat and food intake were right side and left side, respectively. But both sides showed an active role in the response to stress in terms of water intake or plasma glucose. In addition, the effects of chronic stress on hormonal responses included no changes for corticosterone and progesterone levels, while they showed an elevation in estradiol concentration. Moreover, NAc shell has prominent and consistent role in stress effect in right and left sides for corticosterone, in the right side for progesterone, and did not affect estradiol.

In the line with the present study, increased amount of food intake after chronic foot-shock stress in female rats was observed in the previous studies as well [29-31]. Howbeit, Anderson reported that transient stress caused animals to received less food pellets by decreasing lever pressing that consequently led to their weight loss; however, after 14 days of stress, both measures returned to pre-stress levels. On the other hand, in other studies, it was shown that foot-shock stress resulted in decreased food intake in male rats $[32,33]$. In accordance with the current study [34], that was found an increased expression of orexigenic relaxin-3 and misbalanced HPA axis activity raised food intake in condition of chronic stress in female rats [35].Thus, it can be concluded that chronic stress leads to the increment of food intake in female rather than male rats. In the research of Johnson in 2013 it was shown that stress combination with estrogen injection in the ovariectomized female rats leads to increased food intake [36]. It seems that intervention of stress and estradiol caused increased food intake and more considerable CRF gene expression in female in comparison with male rats [37]. This may explain why food intake is different between male and female after chronic stress.

In addition, the inactivation of the right and both sides of NAc shells did not show any statistically significant differences in the amount of food intake in comparison with stressed saline group, while blocking the left side caused a significant increment of food intake, as compared with the saline group. This result shows that the left side of NAc shell may be responsible for the modulatory effect on the increased food intake following chronic stress in female rat but in bilateral interaction left side did not show dominant effect and feeding behavior in bilateral inactivation is in accordance with right side inactivation. Numerous previous research demonstrated that right side of NAc has a stronger role in movement and reward responses to stress compared to left side and there is hemispheric asymmetry in the dopaminergic receptors of this nucleus and these receptors are more concentrated in the right side $[25,38,39]$. In the present study, the role of right NAc in enhancing feeding behavior after chronic stresses induction was proven to be more notable.

In this study, chronic stress resulted in a decreased water intake which was not statistically significant. The Norepinephrine released in stress condition is commonly associated with thirst due to stimulating hypophysal osmo receptor (V3) to secrete vasopressin $[40,41]$. Thirst and increased water intake were observed in the male rats after stress [42], while stress resulted in less water intake in female mice [43]. Cellular organizations in paraventricular nucleus (PVN) are different in female and male rats, as this area contains more CRF neurons in females compared with males. On the other hand, there are more vasopressin-contained neurons in male in comparison with female animals [44].This reason may explain decrement of water intake after chronic stress in female rats in this study. The findings are contrary to the results of other studies which found that stress caused water intake increment in male animals. In addition, the decrease in water intake as result of the inactivation of left, right, and both sides of NAc shells confirms that this area of brain plays an important role in the amount of water intake after stress. This finding is in line with the other study that investigates the key role of NAc in water intake increment in response to stress in female rats [43]. In addition, in this study it revealed that there is no laterality in NAc shell in response to stress and right and left side are involved in increased water intake as the same.

Furthermore, stress was accompanied with anorexia or over eating $[45,46]$ in terms of animal condition or food type. For example, sweet food intake was increased in mice stressed by forceps-squeezed tail [47]. In the previous study, acute stress resulted in an increase in the latency to eat in female rats [24], while, in the present study, the decrease in the latency to eat occurred in chronic stress situation in female animals. Amygdala CRF production and secretion into hypothalamus in response to stress can result in inhibition or stimulation of feeding behaviors in terms of the condition in which animals stay [48,49]. In addition, it is obvious that long term chronic stress causes elevated CRF level in hypothalamus that may consequently result in glucocorticoid elevation. This leads to changes in nutrition pattern and increases in appetite, especially for sweet food, and consequent additional weight gain [50].It has also been elucidated that although elevated cortisol level resulted in weight loss in men, it increased appetite for sweet food in women 
and additional weight gain [51,52]. Therefore, in the present study, the increased appetite and the decrease in the latency to eat can be explained by elevated CRF in the chronic stress situation in female rats. Inactivation of NAcSh sides revealed that the right side had a dominant role in decreasing the latency to eat in the chronic stress. Considering latency to eat as a sign of stress intensity and CRF effects on the anorexia [53], it can be concluded that the right side of NAc shell can play a stronger role in metabolic responses to stress. This finding is in consistence with other researches that showed the importance of right side of NAcSh in the rewarding system and morphine conditioned rats [25].

According to our previous study, similar to acute stress, chronic stress, [24] also increased blood glucose level. Increased blood glucose level in acute stress may be explained by elevated corticosterone, however, in chronic stress, blood glucose level is not relevant to changes of corticosterone, since this hormone level did not change after 4 days of stress in female rats. Nonetheless, it may be related to disrupting mechanism of blood glucose modulation in chronic stress. Similar to our study, Dallman, in 2005, indicated that elevation of glucose concentration in response to chronic stress led to the development of metabolic syndrome, a decreased insulin function, and type II diabetes [54]. In this research according to the previous study [55] the glucose and corticosterone in the male rats increased. Hence, it can be concluded that there are no genderbased differences in this case. Significant elevation of plasma glucose after inactivation of both sides of NAc shell in comparison with sham group, indicated that this nucleus has effective role in the modulation of glucose increment in chronic stress condition and there isn't any laterality in the NAc shell in control of glucose concentration.

Steroid hormone enters cells and binds intracellular receptors to regulate gene expression and cell activities via changing gene profile. These hormones also act by inhibiting other gene regulators that directly contact DNA. In addition, there are many evidences which strengthen the fact that some steroid receptors directly regulate signaling pathways. This finding can be particularly related to the important role of estrogen in the regulation of synapse formation and turnover of prefrontal cortex [56] hypothalamus [57,58] and hippocampus [59].The results of the present study demonstrated that the chronic stress led to significant elevation of plasma estradiol and suggests that the increased amount of this hormone in the chronic stress condition has a determinant role in enhancing cognitive function in female rats via regulating the gene expression in certain regions of the brain while having inhibitory effects in males [59-62]. In present study, inactivation of NAc shell did not cause any significant effect on the estradiol concentration in the chronic stress condition. Whereas, in our previous study, it was revealed that this nucleus has an important role in mediating plasma estradiol concentration in acute stress condition [63]. So, probability adaptation in this nucleus was occurred during time that shift from acute to chronic stress condition and NAc shell lose its regulatory effect on the estradiol concentration during time.

In the previous study, acute stress was proven to increase progesterone and corticosterone levels of blood [24] while, in the present study, chronic stress did not culminate in any changes in both hormones of progesterone and corticosterone levels. This lack of changes may be attributable to the adaptation of animals to chronic stress [26,54]. Inactivation of either left or right sides of NAc indicated significant elevated corticosterone level, while bilateral inactivation did not show a significant elevated corticosterone. With regard to relation of NAc shell with the central nucleus of amygdala in complex of extended amygdala that has an important role in response to stress [64], it seems that transient inactivation of NAcSh destroy the cycle of extended amygdala that resulted in reduction in response to stress and did not display any change in corticosterone level after chronic stress. The effect of transient inactivation of right side and left side of NAcSh that caused significant elevation in corticosterone level shows that this nucleus maybe has modulatory effect on the corticosterone concentration during chronic stress and prevent over increased corticosterone level in the plasma. In addition, significant rise in progesterone level was observed in response to chronic stress when the right side of NAcwas inactivated. Inactivation of the left side or both sides of NAc did not cause any significant changes in progesterone level in response to chronic stress. It seems that the laterality of NAc also diminished in progesterone response to chronic stress due to adaptation comparison with NAc role in acute stress in our previous study [63]. These findings indicate that the right side of NAc shell is effective in adaptation occurrence and decreasing progesterone level in consequence of chronic stress. However, the effect of right side did not show a dominant role in bilateral interaction.

\section{CONCLUSION}

The metabolic and hormonal effects of chronic stress on the female rats are considerable and NAcSh plays an effective role in these responses. Chronic stress leads to increased food intake and the right side of NAcSh caused prominent role in this effect. Also, it showed a decrease in water intake in which both sides 
of NAcSh have the same role. In addition, decreased latency to eat and increased appetite were related to right side of NAcSh while both sides of NAcSh showed modulatory effect on the glucose concentration. Therefore it can be concluded that the right side of NAcSh plays a dominant role in metabolic responses to chronic stress. Right and left side of NAcSh maybe has modulatory effect on the corticosterone level and also right side has modulatory effect on the progesterone level. However, this side could not have a dominant role in bilateral interaction. It can be concluded that the NAc shell plays a pivotal role in metabolic and hormonal responses to chronic stress in a laterality manner in female rats.

Acknowledgments: This work was supported by a Grant from Neuroscience Research Center, Baqiyatallah University of Medical Sciences.

Conflicts of Interest: Declare conflicts of interest or state "The authors declare no conflict of interest."

\section{REFERENCES}

1. Ogawa, N. Psychophysiology of emotion-communication of emotion. Seishinshintaiigaku. 1966;6:352-7.

2. Endo, Y, Shiraki, K. Behavior and body temperature in rats following chronic foot shock or psychological stress exposure. Physiol. \& Behav. 2000; 71: 263-8.

3. Limori, K, Tanaka, M, Kohno, Y, Ida, Y, Nakagawa, R, Hoaki, Y, Tsuda, A, and Nagasaki, N. Psychological stress enhances noradrenaline turnover in specific brain regions in rats. Pharmacol Biochem Behav. 1982; 16: 637-40.

4. Ishikawa, M, Hara, C, Ohdo, S, and Ogawa, N. Plasma corticosterone response of rats with sociopsychological stress in the communication box. Physiol \& Behav. 1992;52:475-80.

5. Ishikawa, M, Ohdo, S, Watanabe, H, Hara, C and Ogawa, N. Alteration in circadian rhythm of plasma corticosterone in rats following sociopsychological stress induced by communication box. Physiol \& Behav. 1995;57:41-7.

6. Jodar, L, Takahashi, M, and Kaneto, $\mathrm{H}$. Effects of footshock-, psychological-and forced swimming-stress on the learning and memory processes: involvement of opioidergic pathways. The Japanese J. Pharmacol. 1995;67:1437.

7. Ogawa, N, Hara, C and Ishikawa, M. Characteristic of socio-psychological stress induced by the communication box method in mice and rats. Environmental stress. 1990;417-27.

8. Sato, Y, Suzuki, N, Horita, H, Wada, H, Shibuya, A, Adachi, H, Tsukamoto, T, Kumamoto, Y, and Yamamoto, M. Effects of Long-term Psychological Stress on Sexual Behavior and Brain Catecholamine Levels. J Androl. 1996;17:83-90.

9. Endo, Y, Yamauchi, K, Fueta, $Y$ and Irie, M. Changes of body temperature and plasma corticosterone level in rats during psychological stress induced by the communication box. Med. Sci. Monit. 2001;7:1161-5.

10. Diamond, D M, Campbell, A, Park, C R, and Vouimba, R. M. Preclinical research on stress, memory, and the brain in the development of pharmacotherapy for depression. Eur Neuropsychopharmacol. 2004;14 Suppl 5:S491-5.

11. McEwen, B S. Central effects of stress hormones in health and disease: Understanding the protective and damaging effects of stress and stress mediators. Eur J Pharmacol. 2008;583:174-85.

12. Oliver, C F, Kutlu, M G, Zeid, D, and Gould, T J. Sex differences in the effects of nicotine on contextual fear extinction. Pharmacol Biochem Behav. 2017.

13. Choudhry, M A, Schwacha, M G, Hubbard, W J, Kerby, J D, Rue, L W, Bland, K I, and Chaudry, I H. Gender differences in acute response to trauma-hemorrhage. Shock. 2005;24 Suppl 1:101-6.

14. Bangasser, D A, and Valentino, R J. Sex differences in molecular and cellular substrates of stress. Cell Mol Neurobiol. 2012;32:709-23.

15. McEWEN, B S, De Kloet, E and Rostene, W. Adrenal steroid receptors and actions in the nervous system. Physiol Rev. 1986;66:1121-88.

16. Campioni, M R, Xu, M, and McGehee, D S. Stress-induced changes in nucleus accumbens glutamate synaptic plasticity. J Neurophysiol. 2009; 101:3192-8.

17. Sousa, N, Cerqueira, J J and Almeida, O F. Corticosteroid receptors and neuroplasticity. Brain research reviews. 2008;57:561-70.

18. Gill, K M and Grace, A A. Differential effects of acute and repeated stress on hippocampus and amygdala inputs to the nucleus accumbens shell. Int J Neuropsychopharmacol. 2013;16:2013-25.

19. Deutch, A Y and Cameron, D S. Pharmacological characterization of dopamine systems in the nucleus accumbens core and shell. Neuroscience. 1992;46:49-56.

20. Heimer, L, Zahm, D, Churchill, L, Kalivas, $P$ and Wohltmann, C. Specificity in the projection patterns of accumbal core and shell in the rat. Neuroscience. 1991;41:89-125.

21. Heinrichs, S C, Menzaghi, F, Pich, E M, Hauger, R L and Koob, G F. Corticotropin-releasing factor in the paraventricular nucleus modulates feeding induced by neuropeptide Y. Brain Res. 1993;611:18-24. 
22. Belcheva, I, Bryer, J B, Starkstein, S E, Honig, M, Moran, T H and Robinson, R G. Hemispheric asymmetry in behavioral response to D 1 and D 2 receptor agonists in the nucleus accumbens. Brain Res. 1990;533:286-91.

23. Belcheva, I, Belcheva, S, Petkov, V and Petkov, V. Asymmetry in behavioral responses to cholecystokinin microinjected into rat nucleus accumbens and amygdala. Neuropharmacology. 1994;33:995-1002.

24. Javadifar, T-S, Sahraei, H, Ketabi, M-A, Nasehi, M and Zarrindast, M-R. Transient inactivation of the nucleus accumbens (NAc) shell prominently ameliorates responses to acute stress in female rats. Brain Res. 2016;1649:18.

25. Esmaeili, M H, Sahraei, H, Ali-Beig, H, Ardehari-Ghaleh, M, Mohammadian, Z, Zardooz, H, Salimi, S H, Shams, J and Noroozzadeh, A. Transient inactivation of the nucleus accumbens reduces both the expression and acquisition of morphine-induced conditioned place preference in rats. Pharmacol Biochem Behav. 2012;102:249-56.

26. Hooshmandi, Z, Rohani, A H, Eidi, A, Fatahi, Z, Golmanesh, L and Sahraei, H. Reduction of metabolic and behavioral signs of acute stress in male Wistar rats by saffron water extract and its constituent safranal. Pharm Biol. 2011;49:947-54.

27. Marcondes, F K, Bianchi, F J and Tanno, A P. Determination of the estrous cycle phases of rats: some helpful considerations. Braz J Biol. 2002;62:609-14.

28. Handa, R J, Burgess, L H, Kerr, J E, and O'Keefe, J A. Gonadal steroid hormone receptors and sex differences in the hypothalamo-pituitary-adrenal axis. Horm Behav.1994;28:464-76.

29. Calvez, J, Timofeeva, E. Behavioral and hormonal responses to stress in binge-like eating prone female rats. Physiol Behav. 2016;157:28-38.

30. Inoue, K, Kiriike, N, Okuno, M, Ito, H, Fujisaki, Y, Matsui, T. Kawakita, Y. Scheduled feeding caused activation of dopamine metabolism in the striatum of rats. Physiol Behav. 1993;53:177-81.

31. Inoue, K, Kiriike, N, Okuno, M, Fujisaki, Y, Kurioka, M, Iwasaki, S, Yamagami, S. Prefrontal and striatal dopamine metabolism during enhanced rebound hyperphagia induced by space restriction--a rat model of binge eating. Biol Psychiatry. 1998;44:1329-36.

32. Samarghandian, S, Ohata, H, Yamauchi, N, Shibasaki, T. Corticotropin-releasing factor as well as opioid and dopamine are involved in tail-pinch-induced food intake of rats. Neuroscience. 2003;116:519-24.

33. Nasihatkon, Z S, Khosravi, M, Bourbour, Z, Sahraei, H, Ranjbaran, M, Hassantash, S M, Sahraei, M, Baghlani, K. Inhibitory effect of NMDA receptors in the ventral tegmental area on hormonal and eating behavior responses to stress in rats. Behav Neurol. 2014;2014:294149.

34. Lenglos, C, Mitra, A, Guevremont, G, Timofeeva, E. Sex differences in the effects of chronic stress and food restriction on body weight gain and brain expression of CRF and relaxin-3 in rats. Genes Brain Behav. 2013;12:37087.

35. Lenglos, C, Mitra, A, Guevremont, G, Timofeeva, E. Sex differences in the effects of chronic stress and food restriction on body weight gain and brain expression of CRF and relaxin-3 in rats. Genes Brain Behav. 2013;12: 370-87.

36. Johnson, Z, Lowe, J, Michopoulos, V, Moore, C, Wilson, M, and Toufexis, D. Oestradiol differentially influences feeding behaviour depending on diet composition in female rhesus monkeys. J Neuroendocrinol. 2013;25:729-41.

37. Handa, R J, Burgess, L H, Kerr, J E, O'Keefe, J A. Gonadal steroid hormone receptors and sex differences in the hypothalamo-pituitary-adrenal axis. Horm behav. 1994;28:464-76.

38. Belcheva, I, Bryer, J B, Starkstein, S E, Honig, M, Moran, TH, Robinson, R G. Hemispheric asymmetry in behavioral response to D1 and D2 receptor agonists in the nucleus accumbens. Brain Res. 1990; 533:286-91.

39. Belcheva, I, Belcheva, S, Petkov, V V, Petkov, V D. Asymmetry in behavioral responses to cholecystokinin microinjected into rat nucleus accumbens and amygdala. Neuropharmacology. 1994;33:995-1002.

40. Danziger, J, and Zeidel, M L. Osmotic homeostasis. Clin J Am Soc Nephrol. 2015;10:852-62.

41. Makara, G B. The relative importance of hypothalamic neurons containing corticotropin-releasing factor or vasopressin in the regulation of adrenocorticotropic hormone secretion. Ciba Found Symp. 1992; 168: 43-51; discussion 51-43.

42. Aguilera, G. HPA axis responsiveness to stress: implications for healthy aging. Exp Gerontol. 2011; 46:90-5.

43. Sarahian, N, Sahraei, H,Zardooz, H,Alibeik, H,Sadeghi, B, Javadifar, TS, Herfehdoost, G R. Comparision of effect of intraperitoneal vs. intra-accumbal injection of memantine on response to acute stress in female NMRI mice. J. Physiol Pharmacol. 2015;18:383-96.

44. Mulder, A H, Geuze, J J, de Wied, D. Studies on the subcellular localization of corticotrophin releasing factor (CRF) and vasopressin in the median eminence of the rat. Endocrinology. 1970;87:61-79.

45. Davis, M, Rainnie, D, and Cassell, M. Neurotransmission in the rat amygdala related to fear and anxiety. Trends Neurosci. 1994;17:208-14.

46. Davis, M, Whalen, P J. The amygdala: vigilance and emotion. Mol Psychiatry. 2001;6:13-34. 
47. O'Doherty, J, Rolls, E T, Francis, S, Bowtell, R, McGlone, F, Kobal, G, Renner, B, Ahne, G. Sensory-specific satietyrelated olfactory activation of the human orbitofrontal cortex. Neuroreport. 2000;11:893-7.

48. Sominsky, L, Spencer, S J. Eating behavior and stress: a pathway to obesity. Front Psychol. 2014;5:434.

49. Stratford, T R, Wirtshafter, D. Injections of muscimol into the paraventricular thalamic nucleus, but not mediodorsal thalamic nuclei, induce feeding in rats. Brain Res. 2013;1490:128-33.

50. Bose, M, Olivan, B, Laferrere, B. Stress and obesity: the role of the hypothalamic-pituitary-adrenal axis in metabolic disease. Curr Opin Endocrinol Diabetes Obes. 2009;16:340-6.

51. Furlan, P M, Ten Have, T, Cary, M, Zemel, B, Wehrli, F, Katz, I R, Gettes, D R, Evans, D L. The role of stressinduced cortisol in the relationship between depression and decreased bone mineral density. Biol Psychiatry. 2005;57:911-7.

52. Habhab, S, Sheldon, J P, Loeb, R C. The relationship between stress, dietary restraint, and food preferences in women. Appetite. 2009;52:437-44.

53. Heinrichs, S C, Menzaghi, F, Pich, E M, Hauger, R L, Koob, G F. Corticotropin-releasing factor in the paraventricular nucleus modulates feeding induced by neuropeptide Y. Brain Res. 1993;611:18-24.

54. Dallman, M F, Pecoraro, N C, la Fleur, S E. Chronic stress and comfort foods: self-medication and abdominal obesity. Brain Behav Immun. 2005;19:275-80.

55. Zardooz, H, Rostamkhani, F, Zaringhalam, J, Shahrivar, F F. Plasma corticosterone, insulin and glucose changes induced by brief exposure to isoflurane, diethyl ether and CO2 in male rats. Physiological Res. 2010;59:973.

56. Hao, J, Rapp, P R, Leffler, A E, Leffler, S R, Janssen, W G, Lou, W, McKay, H, Roberts, J A, Wearne, S L, Hof, P $\mathrm{R}$, Morrison, $\mathrm{J} \mathrm{H}$. Estrogen alters spine number and morphology in prefrontal cortex of aged female rhesus monkeys. J Neurosci. 2006;26:2571-8.

57. Carrer, H F, Aoki, A. Ultrastructural changes in the hypothalamic ventromedial nucleus of ovariectomized rats after estrogen treatment. Brain Res. 1982;240:221-33.

58. Frankfurt, M, Gould, E, Woolley, C S, McEwen, B S. Gonadal steroids modify dendritic spine density in ventromedial hypothalamic neurons: a Golgi study in the adult rat. Neuroendocrinology. 1990;51:530-5.

59. McEwen, B S. Stress, sex, and neural adaptation to a changing environment: mechanisms of neuronal remodeling. Ann N Y Acad Sci. 2010;1204 Suppl:E38-59.

60. Luine, V, Villegas, M, Martinez, C, McEwen, B S. Repeated stress causes reversible impairments of spatial memory performance. Brain Res. 1994; 639: 167-70.

61. Bowman, R E, Zrull, M C, Luine, V N. Chronic restraint stress enhances radial arm maze performance in female rats. Brain Res. 2001; 904: 279-89.

62. Conrad, C D, Grote, K A, Hobbs, R J, Ferayorni, A. Sex differences in spatial and non-spatial Y-maze performance after chronic stress. Neurobiol Learn Mem. 2003;79:32-40.

63. Javadifar, TS, Sahraei, H, Ketabi, M A, Nasehi, M, Zarrindast, M R. Transient inactivation of the nucleus accumbens (NAc) shell prominently ameliorates responses to acute stress in female rats. Brain Res. 2016;1649: 1-8.

64. Koob, GF. The role of the striatopallidal and extended amygdala systems in drug addiction. Ann N Y Acad Sci. 1999;877:445-60. 